\title{
Langerhans Cell Histiocytosis with $B R A F$ p.N486_P490del or MAP2K1 p.K57_G61del Treated by the MEK Inhibitor Trametinib
}

\author{
Yoav Messinger ${ }^{1}$, Bruce Bostrom ${ }^{1}$, Damon Olson ${ }^{1}$, Nathan Gossai ${ }^{1}$, Lane Miller ${ }^{2}$, and \\ Michael Richards ${ }^{1}$ \\ ${ }^{1}$ Children's Hospitals and Clinics of Minnesota \\ ${ }^{2}$ Children's Minneapolis
}

July 28, 2020

\begin{abstract}
Langerhans Cell Histiocytosis (LCH) is characterized by activating variants of the MAPK pathway. Inhibition of the MAPK pathway with trametinib (MEK inhibitor) has been shown to induce responses in LCH patients. Two adolescent males with LCH driven by BRAF p.N486_P490del have received trametinib for $>1$ year with no reactivation in one and partial response in another (including stable lung disease). A third male with neonatal LCH and MAP2K1 p.K57_G61del had a complete response to trametinib with no active disease after 22 months. All patients continue on trametinib monotherapy with tolerable skin and CPK toxicity.
\end{abstract}

\section{Introduction}

The genomic landscape of Langerhans cell histiocytosis (LCH) has been markedly transformed by the identification of variants activating the mitogen-activated protein kinase (MAPK) pathway, the first and most common being the BRAF p.V600E mutation. ${ }^{1}$ In-frame $B R A F$ deletions have subsequently been found in $\mathrm{LCH}$, the most common being the BRAF p.N486_P490 deletion, which was recently detected in 19 of 69 $(28 \%)$ of adults with LCH evaluated by next generation sequencing (NGS). ${ }^{2}$ MAPK pathway inhibition resulted in remarkable responses, but with rapid reactivation upon discontinuation of therapy. ${ }^{3,4}$ Trametinib, a MEK1/2 inhibitor, showed impressive activity in an adult patient reported by Lee et al. ${ }^{5}$ Yet, acquired resistance to trametinib due to MAP2K1 mutations were also recently reported. ${ }^{6}$ Here we are reporting $3 \mathrm{LCH}$ patients withBRAF p.N486_P490del or MAP2K1 p.K57_G61del who are responding to trametinib monotherapy.

\section{Methods and Patients}

This case series was evaluated but the Institutional Review Board (IRB) at Children's Minnesota and deemed exempt from review. Each patient clinical course is summarized in Table 1. Full details of each of the patient is described at length in Supplemental File 1.

Patient 1 is a 15-year-old male who has experienced 5 reactivations of $\mathrm{LCH}$. He presented with left mastoid LCH at 3.5 years of age, confirmed by biopsy with CD1a and S-100 positivity. Reactivations of LCH included the pituitary stalk, mastoid and bone lesions. He was treated with multiple regimens of chemotherapy (Supplemental File 1). Systemic involvement at age 14 included bones, lungs, abdominal lymph nodes, pancreas and perianal area. A course of cladribine, cytarabine and clofarabine resulted in a complete response by PET/CT that was complicated by prolonged bone-marrow suppression. NGS on the last biopsy showed BRAF p.N486_P490 deletion (Fig. 1.A). He was treated with trametinib $0.5-1 \mathrm{mg}$ daily (0.008 - $0.016 \mathrm{mg} / \mathrm{kg}$ ) and remains with no active disease on this dose at 18 months follow-up with intermittent grade 1 skin rash and grade 3 CPK elevation (Table 1 ). 
Patient 2 is 22-year-old male with LCH and 2 reactivations. He was diagnosed at age 18 years with LCH involving lungs, skin and diabetes insipidus. Lung and skin biopsies confirmed LCH with CD1a and S100 positivity. He was treated with cytarabine, but skin reactivation required hydroxyurea and methotrexate. He then had a second reactivation of pulmonary LCH. No biopsy was done but NGS of a prior sample revealed $B R A F$ p.N486_P490 deletion (Fig 1 .A). Therapy with trametinib $2 \mathrm{mg}$ daily $(0.016 \mathrm{mg} / \mathrm{kg}$ ) produced a partial response with residual extensive cystic and nodular lung disease. He continues on monotherapy with trametinib $2 \mathrm{mg}$ daily with stable lung disease after 12 months of therapy. Toxicity has been grade 2 skin rash and grade 4 CPK elevation, but dose level remains unchanged (Table 1).

Patient 3 is a 2-year-old male with multisystem LCH diagnosed at 2 weeks of age when skin biopsy demonstrated CD1a and CD207 positivity. At 2 months of age he had systemic disease including bones, liver, pancreas and lungs. NGS from the skin biopsy revealed a low level of MAP2K1 p.K57_G61del (Fig 1.B). He received a short corticosteroid pulse and trametinib $0.125 \mathrm{mg}(0.030 \mathrm{mg} / \mathrm{kg})$ daily was started in parallel with prednisolone. His lesions rapidly responded and he continues on trametinib monotherapy $0.25 \mathrm{mg}$ daily $(0.019 \mathrm{mg} / \mathrm{kg} /$ day $)$ with no active disease after 22 months of therapy. Toxicity has been a grade 1 skin rash and grade $1 \mathrm{CPK}$ elevation (Table 1 ).

\section{Discussion}

This series of patients illustrates both the promise and limitation of MEK inhibition with trametinib monotherapy for LCH. Specifically, patient 3 with MAP2K1 deletion and systemic LCH in the newborn period showed a complete and durable response to trametinib and is thriving with no active disease. On the other hand, patient 2 with $B R A F$ deletion has had only a partial response to full dose trametinib.

As suggested by Allen et al., there is a significant difference between infants with isolated cutaneous LCH and infants who develop progressive, life-threatening disease as patient $3 .{ }^{1}$ The misguided myeloid differentiation model proposed by Allen et al. would suggest that patient 3 developed MAP2K1 deletion early in fetal life, possibly in the yolk-sac progenitors or fetal liver monocytes. ${ }^{1}$

The misguided myeloid differentiation model also suggests that the origin of neurodegenerative LCH disease is from a hematopoietic cell clone. ${ }^{1}$ Whether this is true only for BRAFp.V600E mutation or other MAPK pathway variants remains to be seen. ${ }^{7,8}$ So far none of our patients have shown neurodegenerative changes. McClain et al demonstrated BRAFp.V600E in peripheral mononuclear cells in patients with neurodegenerative LCH. ${ }^{8}$ We did not have the capacity to evaluate the presence of the variants reported here in the peripheral blood of our patients.

Though most patients with pulmonary LCH are adults with a smoking history, our patients are younger $(2$ months -18 years) with no smoking history. ${ }^{4}$ Jouenne et al., in a large adult LCH group, noted the BRAF N486_P490del as the second most common variant after BRAF V600E mutation. This was especially seen in association with lung disease. ${ }^{2}$

The dose and length of therapy of trametinib needed to inhibit the MAPK pathway in LCH is not established. A retrospective study of 21 pediatric LCH patients with MAPK pathway somatic mutation (BRAF p.V600E, $\mathrm{n}=20 ; M A P 2 K 1 c .293 \_310 \mathrm{del}, \mathrm{n}=1$ ) who received MAPK pathway inhibitors after failure of at least 1 prior therapy was recently reported by the NACHO-LIBRE Study Group. ${ }^{9}$ They reported $86 \%$ response rate to therapy, and 6 patients in that group received trametinib either in combination with BRAF inhibitors or alone. The dose of trametinib in that report ranges from $0.0125-0.018 \mathrm{mg} / \mathrm{kg}$ or reported as $1 \mathrm{mg}$ or $2 \mathrm{mg}$ daily. Patient 21 in that report had an identical MAP2K1 deletion to our patient number 3. That patient had brief response to trametinib for 2 months before suffering progressive disease. ${ }^{9}$ In contrast, our patient 3 had remarkable response to trametinib daily lasting almost 2 years. Lee et al. described a 36 year old female with similar BRAF p.N486_P490 deletion to our patients 1 and 2 who had a remarkable response to trametinib within 5 days including PET/CT negativity after 4 months and elimination of seizures within weeks. ${ }^{5}$ The dose of trametinib was not reported. Notably, our patient 2 had partial response of lung disease on monotherapy with trametinib $2 \mathrm{mg}$ daily. 
Diamond et al. report that the MEK1/2 inhibitor cobimetinib (60 mg daily for 21 days of a 28-day cycle) resulted in remarkable response rate (89\%) among adults with histiocytic disorders. ${ }^{10}$ In that report a patient with LCH and BRAF N486_P490del did seem to have a complete response lasting 2 years, as did some of the patients with MAP2K1 mutations. It is certainly possible that cobimetinib is a better MEK inhibitor for $\mathrm{LCH}$

In summary, $3 \mathrm{LCH}$ patients are currently receiving monotherapy with trametinib for systemic LCH. Two have BRAF p.N486_P490del and one has MAP2K1 p.K57_G61del. These patients are tolerating therapy with low grade skin toxicity and tolerable elevations in CPK levels. Two (patients 1 \& 3) are maintaining non-active disease (follow-up 18-22 months) and one (patient 2) who had partial response is stable with active disease (follow-up 12 months).

\section{References}

1. Allen CE, Merad M, McClain KL. Langerhans-Cell Histiocytosis. N Engl J Med. 2018;379(9):856-868.

2. Jouenne F, Chevret S, Bugnet E, et al. Genetic landscape of adult Langerhans cell histiocytosis with lung involvement. Eur Respir J. 2020;55(2):1901190.

3. Donadieu J, Larabi IA, Tardieu M, et al. Vemurafenib for Refractory Multisystem Langerhans Cell Histiocytosis in Children: An International Observational Study. J Clin Oncol. 2019;37(31):2857-2865.

4. Rodriguez-Galindo C, Allen CE. Langerhans cell histiocytosis.Blood. 2020;135(16):1319-1331.

5. Lee LH, Gasilina A, Roychoudhury J, et al. Real-time genomic profiling of histiocytoses identifies earlykinase domain BRAF alterations while improving treatment outcomes. JCI Insight.2017;2(3):e89473.

6. Azorsa DO, Lee DW, Wai DH, et al. Clinical resistance associated with a novel MAP2K1 mutation in a patient with Langerhans cell histiocytosis.Pediatr Blood Cancer. 2018;65(9):e27237.

7. Héritier S, Barkaoui MA, Miron J, et al. Incidence and risk factors for clinical neurodegenerative Langerhans cell histiocytosis: a longitudinal cohort study. Br J Haematol. 2018;183(4):608-617.

8. McClain KL, Picarsic J, Chakraborty R, et al. CNS Langerhans cell histiocytosis: Common hematopoietic origin for LCH-associated neurodegeneration and mass lesions. Cancer.2018;124(12):2607-2620.

9. Eckstein OS, Visser J, Rodriguez-Galindo C, Allen CE, Group N-LS. Clinical responses and persistent BRAF V600E $(+)$ blood cells in children with LCH treated with MAPK pathway inhibition Blood 2019; 15:1691-1694.

10. Diamond EL, Durham BH, Ulaner GA, et al. Efficacy of MEK inhibition in patients with histiocytic neoplasms. Nature.2019;567(7749):521-524.

11. Chen SH, Zhang Y, Van Horn RD, et al. Oncogenic BRAF Deletions That Function as Homodimers and Are Sensitive to Inhibition by RAF Dimer Inhibitor LY3009120. Cancer Discov. 2016;6(3):300-315.

12. Rollins BJ. Biology and Genomics of LCH and Related Disorders. In: Abla O, Janka G, eds. Histiocytic disorders. Cham, Switzerland: Springer; 2018:53-71.

\section{Figure 1 Legend:}

Variant protein linear diagrams: (A) Patients 1 and 2 were found to have 15-base pair in-frame deletions in exon 12 of BRAF, resulting in a predicted deletion of five amino acids from the protein kinase domain of the protein. This area represents the $\beta 3 / \alpha \mathrm{C}$-helix loop of the kinase domain and is an important structural region allowing for conformational changes between the $\alpha \mathrm{C}$-helix-in and $\alpha \mathrm{C}$-helix-out conformations. In-frame deletions in this area have been shown by molecular modeling to shorten the loop, locking the $\alpha \mathrm{C}$-helix in the $\alpha \mathrm{C}$-helix-in conformation which favors BRAF protein dimerization and activation of the downstream MAPK pathway. The most common LCH pathogenic variant, p.V600E, is shown for reference. ${ }^{11}$ (B) Patient 3 had a 15-base pair in-frame deletion in exon 2 of MAP2K1, resulting in a predicted deletion of five amino acids 
from the N-terminal negative regulatory domain of the protein which presumably derepresses the kinase domain, leading to subsequent MAPK pathway activation. ${ }^{12}$

Conflict of interest: The authors report no conflict of interest.

Acknowledgements: The authors thank the generosity of the patients and their family who agreed to share their information with the public.

Source of funding: no source of funding was available for this report.

\section{Hosted file}

Trametinib Table 1 7-27-20.docx available at https://authorea.com/users/346723/articles/ 472618-langerhans-cell-histiocytosis-with-braf-p-n486_p490del-or-map2k1-p-k57_g61del-

treated-by-the-mek-inhibitor-trametinib

Figure 1

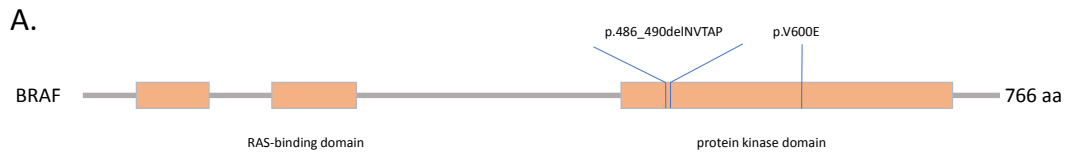

B.

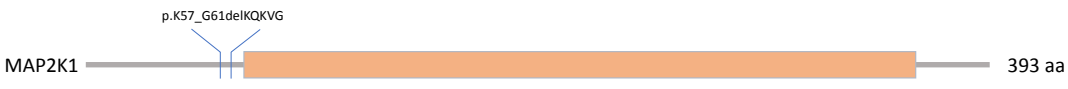

protein kinase domain 